\title{
Some roles of the vocal tract in clarinet breath attacks: Natural sounds analysis and model-based synthesis
}

\author{
Philippe Guillemain ${ }^{\mathrm{a})}$ \\ Laboratoire de Mécanique et d'Acoustique, CNRS UPR 7051 \\ 31 Chemin Joseph Aiguier, 13402 Marseille Cedex 20, France
}

(Dated: January 16, 2007)

\begin{abstract}
A simplified physical model mainly devoted to the reproduction of some transients of clarinetlike instruments is presented. From time-frequency analyses of natural clarinet sounds, it is shown that the vocal tract can play a significant role in some attacks as well as in the permanent regime. The model proposed consists in supplying a pressure source at the entrance of a cylindrical bore attached to the mouthpiece, allowing to reach various vocal tract configurations. For real-time synthesis purposes, a digital scheme solving the physical problem is proposed. It is shown that this synthesis model is able to reproduce some of the complex features observed during the attacks of the natural sounds analyzed, as well as known effects of the vocal tract in permanent regime.
\end{abstract}

PACS numbers: 43.75.Ef, 43.75.Pq

\section{INTRODUCTION}

Synthesis models based upon simplified representations of the physical functioning of reed instruments have shown their ability to produce musically relevant timbre variations with respect to commands of the player. Moreover, such models are interesting from a real-time control point of view, since they minimize the mapping between the continuous command of the player and the synthesis parameters. In counterpart, direct measurement of controls on a player in performance situation is a task difficult to handle and the complex mechanisms responsible for the sound production make difficult to know what the musician is doing from the analysis of the sound he produces. Moreover, inverting a model requires a deep but generally unavailable knowledge of some of its parts, such as the whole resonator. Indeed, though Backus ${ }^{1}$ considered the vocal tract influence of the player to be negligible, it is now commonly stated that the resonator of reed instruments should be seen as an association of two coupled acoustic bores in series, one corresponding to the instrument and the other to the player (see e.g. Benade $^{2}$ ).

Sommerfeldt and Strong ${ }^{3}$ and more recently Fritz ${ }^{4}$ have studied experimentally and numerically, out of the real-time synthesis context, how the impedance peaks of the vocal tract may modify some features of the clarinet sound (spectrum, playing frequency). Scavone ${ }^{5}$ proposed a real-time oriented synthesis model and discussed in another paper ${ }^{6}$ several "physically informed" attack models including noisy components.

While these authors focus on the role of the vocal tract,

a) Electronic address: guillem@lma.cnrs-mrs.fr modelled for some of them ${ }^{3,5}$ with analogous electric circuits, during the permanent regime, the present paper investigates vocal tract influences during breath attacks without mechanical action on the reed and proposes a highly simplified distributed model for real-time sound synthesis. The aim of this paper is not to present a systematic study of vocal tract manipulations used in musical performances and their consequences on the sound produced but rather to use the classical "analysis by synthesis"7 framework to propose a model capable to mimic natural sounds displaying specific features and to determine the corresponding set of parameters relevant both from the physical and the synthesis points of view.

After a brief recall of Fritz's ${ }^{4}$ model, section II proposes a model in which the pressure is supplied at the entrance of an upstream bore rather than at the reed level. For sake of simplicity, this upstream bore is considered cylindrical and intends to provide a model of the first impedance peak of the whole respiratory airway.

In section III, time-frequency analyses of transients of natural clarinet sounds are presented and hypotheses are made to explain the complex behaviors observed during the attacks.

Thanks to the presentation of a synthesis scheme that simulates the behavior of the physical model, section IV shows that during attack transients, when the blowing pressure is supplied at the entrance of the upstream bore, self-oscillations tuned on its first impedance peak may start and decrease until the steady-state regime tuned on the instrument bore is reached.

It is concluded that the synthesis model generates transient and permanent regimes sharing many common features with the natural sounds analyzed in section III. 


\section{PHYSICAL MODEL}

The classical model describing the vocal tract by its impedance seen from the reed is first briefly recalled.

\section{A. Classical model}

The classical model (see e.g Wilson and Beavers ${ }^{8}$ ) used to represent the link between acoustic pressure and flow in the mouthpiece of a single reed instrument is based upon the steady Bernoulli equation. It is assumed that a jet of velocity $v_{j}(t)$ and pressure $p_{j}(t)$ is formed at the end of the reed channel, and that its kinetic energy is totally dissipated (in the mouthpiece for a jet entering the resonator, in the mouth for a jet entering the player's vocal tract). It is also assumed that the cross-section $S_{j}(t)$ of this jet is much smaller than the mouth crosssection $S_{m}$ and the resonator cross-section $S$. It is finally assumed that the jet cross-section is proportional to the time varying reed channel opening $S_{y}(t)$, determined by the reed motion: $S_{j}(t)=\alpha S_{y}(t)$. For simplicity, it will be assumed in what follows that $\alpha=1$.

With these hypotheses:

$$
p_{m}-p_{r}(t)=\frac{1}{2} \rho \frac{u_{r}(t)\left|u_{r}(t)\right|}{S_{y}(t)^{2}}
$$

where $\rho$ is the mean air density. $p_{m}$ is the mouth pressure. $p_{r}(t)$ and $u_{r}(t)$ are respectively the acoustic pressure and flow at the entrance of the resonator.

In permanent regime, by decomposing the mouth pressure $p_{m}$ into an imposed static (DC) component $p_{0}$ and an oscillating component $p_{u}(t)$ created by the acoustic coupling between the vocal tract and the body of the instrument, Fritz ${ }^{4}$ studied the self-oscillations by considering an equivalent impedance at the reed level including the upstream and downstream bores:

$$
Z_{\text {tot }}(\omega)=\frac{P_{r}(\omega)-P_{u}(\omega)}{U_{r}(\omega)}=Z_{r}(\omega)+Z_{u}(\omega)
$$

where the capital letters denote the Fourier transforms. $Z_{u}(\omega)=-P_{u}(\omega) / U_{r}(\omega)$ and $Z_{r}(\omega)=P_{r}(\omega) / U_{r}(\omega)$ denote the impedances of the upstream and downstream bores.

Nevertheless, this model can be questionable from a physical point of view in the case of a time varying blowing pressure, since the static pressure $p_{0}$ is imposed at the entrance of the reed channel. This makes the mouth pressure depend only on the acoustic coupling between the two bores and on the imposed pressure but not on the vocal tract itself, independently of the instrument.

\section{B. Proposed model}

We propose a model in which the mouth pressure $p_{m}$, from now denoted $p_{m}(t)$, is the consequence of a blowing pressure $p_{g}(t)$ imposed at the entrance of an upstream

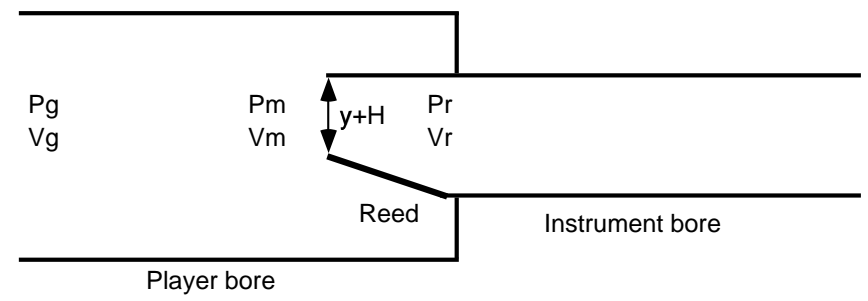

FIG. 1. Geometry of the system and physical variables.

bore surrounding the mouthpiece. Though Scavone ${ }^{5}$ considered an equivalent lung flow source, simple calculations based on measurements by Mukai ${ }^{9}$ show that in permanent regime, the cross-section area of the glottis is about three times larger for experienced musicians and twenty times larger for inexperienced musicians than the average reed channel opening of a clarinet. Moreover, during attacks the energy source can be located at the palatal constriction level rather than at the glottis or lungs level. For these reasons, supplying a pressure seems as realistic as supplying a flow and will be more convenient for real-time synthesis purposes.

\section{Resonator model}

The geometry of the system and its physical variables are depicted in figure (1).

The body of the instrument is assumed to be a perfect cylinder of equivalent (including only the imaginary part of the radiation impedance ${ }^{10}$ as a length correction) length $L$ and radius $R$, with linear propagation of plane waves. In this case, its input impedance linking the Fourier transforms of the pressure $p_{r}(t)$ and flow $u_{r}(t)$ in the mouthpiece of the instrument is:

$$
Z_{r}(\omega)=j Z_{c} \tan (k(\omega) L)
$$

$Z_{c}=\rho c / S$ is the characteristic impedance of the bore. $S$ is its input cross-section. $k(\omega)$ is the wavenumber including viscothermal losses ${ }^{11}: k(\omega)=\omega / c-j^{3 / 2} \eta c \sqrt{\omega}$, where $\eta=1 /\left(R c^{3 / 2}\right)\left(\sqrt{l_{v}}+\left(c_{p} / c_{v}-1\right) \sqrt{l_{t}}\right)$. Typical values of the physical constants, in mKs units, are: $c=340$, $l_{v}=4.10^{-8}, l_{t}=5.6 .10^{-8}, c_{p} / c_{v}=1.4$.

For sake of simplicity, digital efficiency and controllability issues in a real-time synthesis context, the "player bore" is also considered cylindrical, with radius $R_{m}$, cross-section $S_{m}$ and length $L_{m}$. It includes frequency dependent losses and models the low frequency behavior of the vocal tract. The losses model, taken into account in the wavenumber denoted $k_{m}$, is also based upon the classical viscothermal losses rule ${ }^{11}$ but the geometrical radius is replaced by a smaller and adjustable radius $R_{p}$ in order to take into account additional losses corresponding to those of the human tissues. Indeed, a straightforward simulation shows that this method provides an approximation of Sondhi's ${ }^{12}$ losses model sufficient for digital 
sound synthesis purposes. Moreover, it provides an additional degree of freedom to control the quality factor of the first impedance peak independently of the bore length and radius. For digital synthesis purposes, it is finally assumed that $k_{m}$ equals zero at zero frequency. This way, the $\mathrm{DC}$ component of the blowing pressure $p_{g}(t)$ is fully transmitted to the mouth pressure $p_{m}(t)$.

Obviously, in real situations, these parameters are modified dynamically by the player during the attack and during the play, but for the purpose of this paper which mainly aims at understanding and simulating the role of the vocal tract during the transient, it will be assumed that they remain constant. The three control parameters $S_{m}, L_{m}$ and $R_{p}$ allow to reach different configurations, running from the classical model in which $S_{m}$ is large and $L_{m}$ is small, to more realistic configurations in which $L_{m}$ and $R_{p}$ will determine respectively the frequency and the quality factor of the first impedance peak of the vocal tract and $S_{m}$ will determine the level of acoustic coupling between the player bore and the instrument bore.

By denoting $U_{m}=S_{m} V_{m}$ and $U_{g}=S_{m} V_{g}$ the Fourier transforms of the acoustic flows associated to the acoustic velocities $v_{m}$ and $v_{g}$, the pressure and flow propagation within the player bore is described by the following transmission line equations:

$$
\begin{aligned}
& P_{g}(\omega)=\cos \left(k_{m} L_{m}\right) P_{m}(\omega)+j Z_{m} \sin \left(k_{m} L_{m}\right) U_{m}(\omega) \\
& U_{g}(\omega)=\frac{j}{Z_{m}} \sin \left(k_{m} L_{m}\right) P_{m}(\omega)+\cos \left(k_{m} L_{m}\right) U_{m}(\omega)
\end{aligned}
$$

where $Z_{m}=\rho c / S_{m}$ is the characteristic impedance of the upstream bore.

These equations are finally written as follows:

$$
\begin{array}{r}
P_{g}(\omega)=Z_{m} U_{g}(\omega)+e^{-j k_{m} L_{m}}\left(P_{m}(\omega)-Z_{m} U_{m}(\omega)\right)(4) \\
P_{m}(\omega)=-Z_{m} U_{m}(\omega)+e^{-j k_{m} L_{m}}\left(P_{g}(\omega)+Z_{m} U_{g}(\omega)\right)(5)
\end{array}
$$

It is worth noting that since $P_{g}(\omega)$ is imposed, equations (4) and (5) can be combined to remove $U_{g}(\omega)$ :

$$
P_{m}(\omega)=\frac{2 e^{-j k_{m} L_{m}}}{1+e^{-2 j k_{m} L_{m}}} P_{g}(\omega)-j Z_{m} \tan \left(k_{m} L_{m}\right) U_{m}(\omega)
$$

which shows that the resonances induced by the vocal tract are those of a quarter wave resonator. In permanent regime $\left(P_{g}(\omega)=p_{0} \delta(\omega)\right)$, this model is equivalent to Fritz's ${ }^{4}$ model. As soon as $p_{g}(t)$ varies, the two models are different, due to the filtering of the blowing pressure by $2 \exp \left(-j k_{m} L_{m}\right) /\left(1+\exp \left(-2 j k_{m} L_{m}\right)\right)$. Therefore, during attack transients, it can be expected that a large bandwidth excitation yields oscillations of $p_{m}(t)$ tuned on the poles of this filter, independently of the level of the acoustic coupling between the two bores occurring in permanent regime and determined by the value of the impedance $j Z_{m} \tan \left(k_{m} L_{m}\right)$.

\section{Flow model}

If it is no longer assumed that the jet cross-section is much smaller than the mouth cross-section, the acoustic velocity $v_{m}(t)$ in the player bore can no longer be ignored. In this case, for a jet entering the instrument, the Bernoulli flow model reads:

$$
p_{m}(t)+\frac{1}{2} \rho v_{m}(t)^{2}=p_{j}(t)+\frac{1}{2} \rho v_{j}(t)^{2}
$$

Assuming flow conservation $\left(u_{m}(t)=S_{m} v_{m}(t)=u_{r}(t)=\right.$ $\left.S_{y}(t) v_{j}(t)\right)$ and total dissipation of the kinetic energy of the jet in the mouthpiece $\left(p_{r}(t)=p_{j}(t)\right)$, equation $(7)$ becomes in terms of pressure and flow variables:

$$
p_{m}(t)-p_{r}(t)=\frac{1}{2} \rho u_{r}(t)^{2}\left(\frac{1}{S_{y}(t)^{2}}-\frac{1}{S_{m}^{2}}\right)
$$

In the same way, for a jet entering the mouth, the Bernoulli flow model reads:

$$
p_{r}(t)+\frac{1}{2} \rho v_{r}(t)^{2}=p_{j}(t)+\frac{1}{2} \rho v_{j}(t)^{2}
$$

Assuming again flow conservation and total dissipation of the kinetic energy of the jet in the mouth $\left(p_{m}(t)=\right.$ $p_{j}(t)$ ), equation (9) becomes in terms of pressure and flow variables:

$$
p_{m}(t)-p_{r}(t)=-\frac{1}{2} \rho u_{r}(t)^{2}\left(\frac{1}{S_{y}(t)^{2}}-\frac{1}{S^{2}}\right)
$$

The reed channel opening $S_{y}(t)$ is determined by the product of its width $w$ and its height $H+y(t)$ (see figure (1)):

$$
S_{y}(t)=w \theta(H+y(t))(H+y(t))
$$

where $\theta(H+y(t))$ is the Heaviside function, the role of which is to keep the reed channel opening positive $(\theta(H+$ $y(t))=0$ when $y(t) \leq-H)$ and to model the beatingreed phenomenon. $H$ denotes the position of the reed at equilibrium (without any blowing pressure).

The reed is modelled as a linear single degree of freedom system and its displacement $y(t)$ is given by the following dynamic equation:

$$
\frac{1}{\omega_{r}^{2}} \frac{d^{2} y(t)}{d t^{2}}+\frac{q_{r}}{\omega_{r}} \frac{d y(t)}{d t}+y(t)=-\frac{p_{m}(t)-p_{r}(t)}{\mu_{r} \omega_{r}^{2}}
$$

where $\omega_{r}=2 \pi f_{r}, q_{r}^{-1}$ and $\mu_{r}$ are respectively the angular frequency, the quality factor of the reed resonance and the reed mass per unit area.

The full physical model of the functioning of the instrument is made of:

- The blowing pressure $p_{g}(t)$.

- The equations (4) and (5) describing the propagation of pressure and flow between each termination of the player bore.

- The instrument bore impedance equation (3).

- The reed dynamics equation (12).

- The reed channel opening equation (11).

- The flow model equations (8) and (10) . 


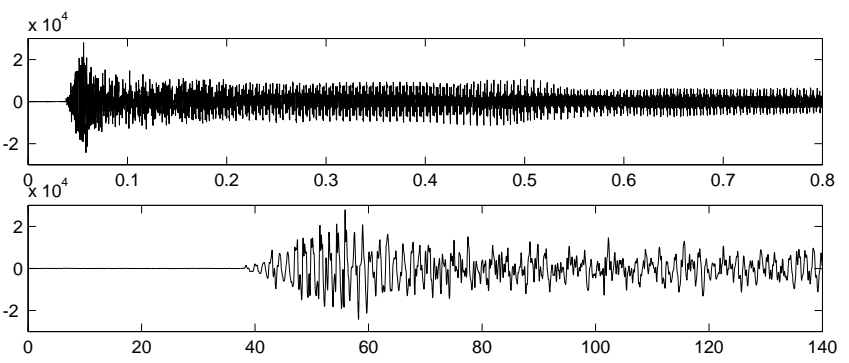

FIG. 2. Example 1. Top: First $0.8 s$ of the time signal. Bottom: Enlargement of the first $140 \mathrm{~ms}$.

\section{EXPERIMENTAL OBSERVATIONS}

This section firstly presents time-frequency analyses of transient parts of two natural clarinet sounds. Then, it proposes an interpretation of the phenomena, linked with general features of the impedance (seen from the reed) of the vocal tract, based on measurements by Fritz $^{4}$ and simulations by Sommerfeldt ${ }^{3}$.

\section{A. Analysis of natural sounds}

In order to study the role of the player bore during transients, two musicians were asked to play attacks without involving a mechanical contact between the tongue and the reed. Example 1 was performed by an inexperienced player. Example 2 was played by an experienced clarinet teacher and performer on its own instrument. This musician pays a lot of attention to what he calls his "internal phonation" and is, according to him, trained to control the movements and shape of its respiratory airway when playing.

All the spectrograms and spectrogram slices presented in what follows have been computed with a gaussian window, the width of which at half-height is $25 \mathrm{~ms}$.

\section{Example 1}

The top of figure (2) shows the external pressure, recorded at a sampling frequency of $44.1 \mathrm{kHz}$ one meter away from the instrument with an omnidirectional microphone, of the first $0.8 s$ of an attack of a clarinet sound. The bottom shows an enlargement of the first $140 \mathrm{~ms}$. The fundamental frequency, estimated in the steady-state part of the sound, is $149 \mathrm{~Hz}$. The envelope of the attack shows an increasing phase, followed by a decreasing phase. The total duration of these two phases is about $40 \mathrm{~ms}$. The main frequency of oscillation of the signal during these phases is around $700 \mathrm{~Hz}$ and is far above the fundamental frequency of the steady-state regime.

Figure (3) shows the spectrogram of the sound in the range $[04 k \mathrm{~Hz}$ ]. During the first $0.1 \mathrm{~s}$, the component of highest level is a transient component of short duration (around $40 \mathrm{~ms}$ ), the frequency of which is around $690 \mathrm{~Hz}$. This frequency does not correspond to the frequency of one of the harmonics of the sound (less than

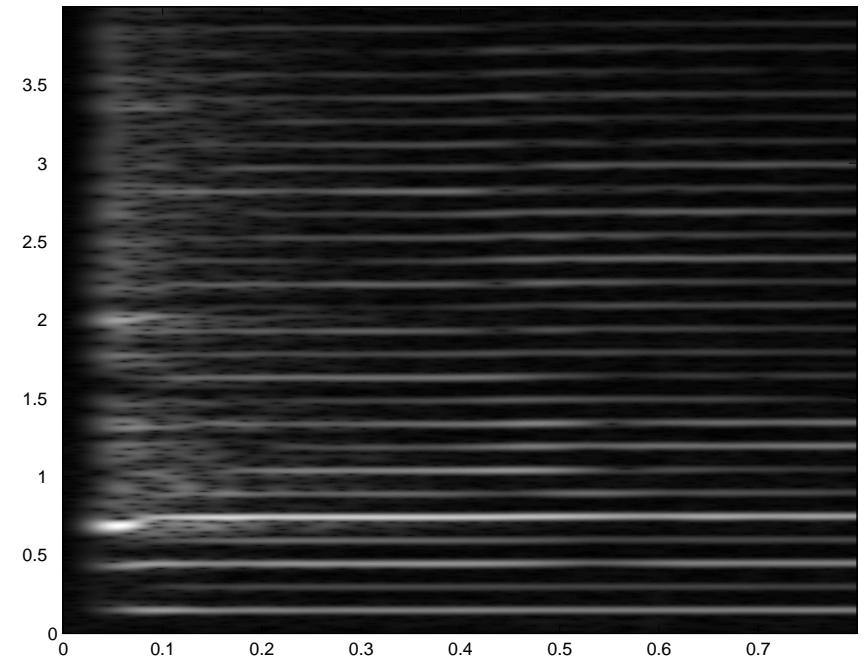

FIG. 3. Example 1. Spectrogram. Horizontal axis in seconds, vertical axis in $\mathrm{kHz}$.

the frequency of the $5^{\text {th }}$ harmonic) and the spectrogram does not show a smooth glissando between the frequency of this transient component and that of the fifth harmonic. This transient component appears before the beginning of the fifth harmonic and dies after it. One can notice around $2 \mathrm{kHz}$ a similar component at a lower level. Its frequency corresponds to three times that of the first transient component. In the same way, it can be noticed around $3.4 \mathrm{kHz}$ a transient component whose frequency corresponds to five times that of the main transient component. After the first $0.1 s$, the fifth harmonic has the highest level. During the first $0.2 s$, the spectral content of the sound is rich, with the presence of many components that seem to be neither in harmonic relationships between them nor subharmonic of the fundamental frequency. During the first $0.5 \mathrm{~s}$, the amplitudes of the harmonics vary rapidly, which seems to indicate that a stable, steady state oscillation regime is not reached.

This is confirmed by figure (4), which shows superimposed two vertical "slices" of the spectrogram at $t=$ $0.07 \mathrm{~s}$ (solid line) and $t=0.7 \mathrm{~s}$ (dashed line). For clarity the curves have been normalized. The spectrogram slice corresponding to the attack shows clearly the component at $690 \mathrm{~Hz}$, as well as its harmonics. Harmonic two emerges among other peaks. Harmonic three is clearly visible around $2 \mathrm{kHz}$. Harmonic four does not emerge and harmonic five is split into two components around $3.4 \mathrm{kHz}$. All these transient components are no longer visible on the slice corresponding to the permanent regime and their frequencies are different from those of the stable self-oscillations. In permanent regime, harmonics three and five have a higher level than the fundamental.

It is worth noting that other instances of the same note played by the same musician lead to similar results. It has been noticed that notes played with a lower blowing 


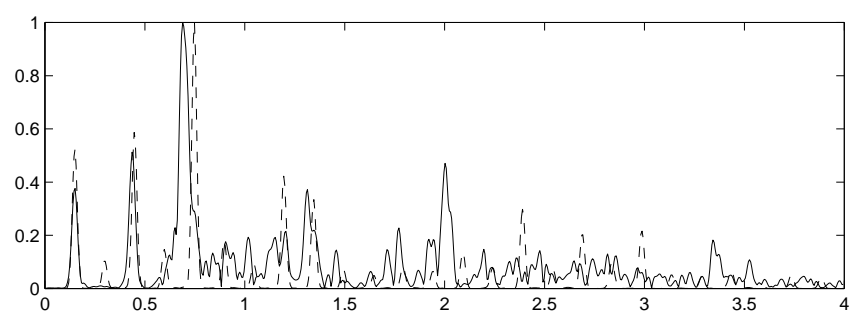

FIG. 4. Example 1. Spectrogram slices at $t=0.07 \mathrm{~s}$ (solid line) and $t=0.7 \mathrm{~s}$ (dashed line). Horizontal axis in $\mathrm{kHz}$.
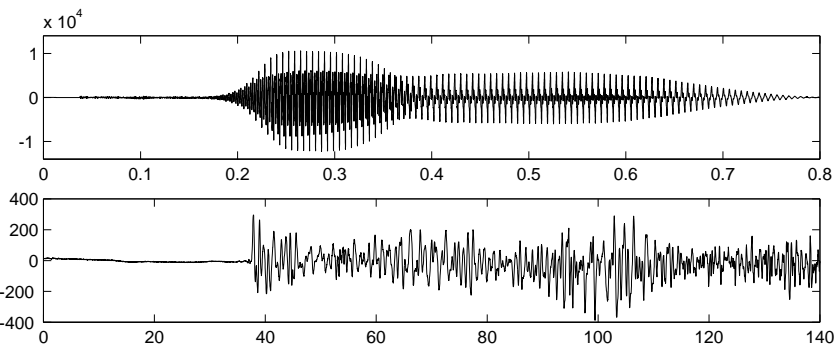

FIG. 5. Example 2. Top: First $0.8 s$ of the time signal. Bottom: Enlargement of the first $140 \mathrm{~ms}$.

pressure lead to the death of the harmonics of the transient component at $690 \mathrm{~Hz}$ and to the simultaneous birth of the transient component and the fifth harmonic of the steady-state regime. In the same way, a different note (two semitones higher) played by the same musician on a different clarinet lead to a qualitatively similar behavior of the spectrogram during the transient, with the presence of a transient component around $700 \mathrm{~Hz}$ and a rich spectral content during the first $0.1 \mathrm{~s}$.

\section{Example 2}

Figure (5) shows that in this example, the attack is slower than in the first example. Self-oscillations start around $t=0.2 \mathrm{~s}$. The enlargement shows that during the beginning of the transient, mainly noise seems to be present.

Figure (6) shows that the brightness of the sound is weaker than in the first sound example by considering the smaller level of high frequency harmonics in the steady state part of the sound. Between 0 and $1200 \mathrm{~Hz}$, all the harmonics appear nearly simultaneously, excepted harmonic three, appearing before the others, in the continuation of a noisy and low level transient component at $500 \mathrm{~Hz}$. The levels of harmonics three and five are clearly higher than that of the fundamental. Similarly, harmonics seven and eight also have a high level. From the beginning of the self-oscillations until $t=0.35 \mathrm{~s}$, the components are frequency modulated and seem to remain in harmonic relationships. An inharmonicity in the whole resonator might be the responsible for this pitch variation. In the steady state regime, above the odd har-

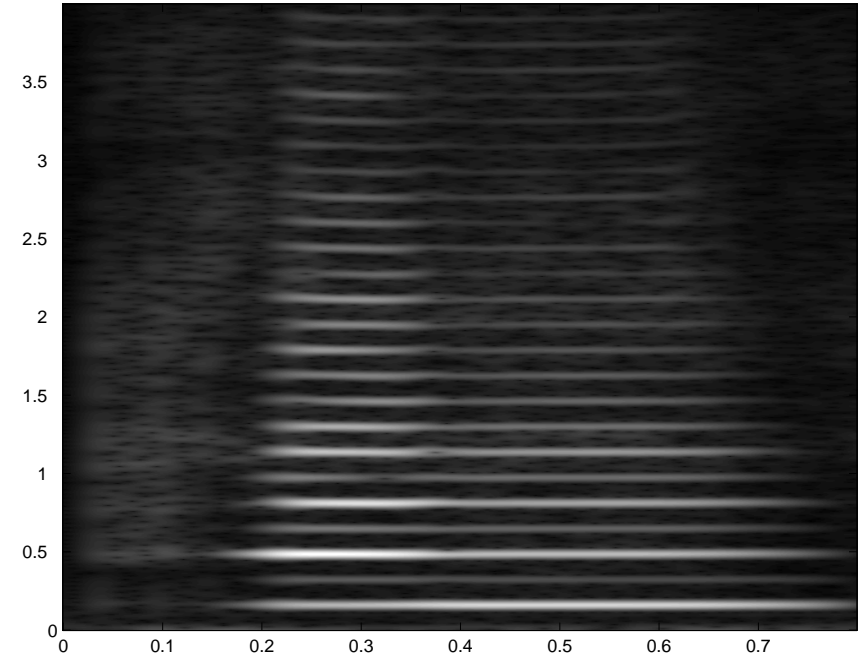

FIG. 6. Example 2. Spectrogram. Horizontal axis in seconds, vertical axis in $k H z$.

monic number seven (around $1200 \mathrm{~Hz}$ ), the level of the even harmonics is high.

It is worth noting that a different note played by the same musician (ten semitones higher) also exhibits an early start of a high level third harmonic in the continuation of a transient component whose frequency is close to that of the third harmonic (around $850 \mathrm{~Hz}$ ).

\section{B. Discussion}

According to musicians and measurements by Fritz, ${ }^{4}$ two main vocal tract configurations are used in permanent regime.

The / a/ configuration (as in "father") is used in the lower register. In this configuration, the impedance of the player air column seen from the reed shows a peak around $300-500 \mathrm{~Hz}$. The height of this peak remains small compared to those of the instrument bore.

The / $i$ / configuration (as in "see") is preferably used in the higher register. In this configuration, the impedance of the player air column seen from the reed shows a peak around $700-900 \mathrm{~Hz}$. The height of this impedance peak is of the same order of magnitude as those of the instrument bore.

As suggested by Clinch ${ }^{13}$ and measured by Wilson, ${ }^{14}$ a trained player may align the first resonance of his vocal tract to an harmonic of the note played. This could correspond to the second sound example. Indeed, this sound is played by an experienced teacher, in the lower register. Thus, it can be hypothesized that the high level of harmonic three corresponds to an alignment of a resonance of the vocal tract in a configuration close to / $a /$ with a resonance of the instrument and that a strong coupling of the two bores at this frequency is responsible for the early birth of the third harmonic. Another argument in favor of this hypothesis is that a different note played by 
this musician shows a similar behavior.

On the contrary, the performer of the first example did not make any matching between his vocal tract configuration and the note played. The high level of harmonic five seems to indicate that he used a vocal tract configuration closer to $/ i /$ than to $/ a /$, with a strong resonance located between the harmonics four and five. Moreover, he supplied the pressure suddenly at a high level. Therefore, it can be hypothesized that a mechanism involving the vocal tract is responsible for the raising of a distinct transient component around $690 \mathrm{~Hz}$ accompanied by its harmonics and many inharmonic components. These hypotheses are sustained by the fact that a different note played by the same player on a different instrument exhibits a similar transient component at roughly the same frequency.

In order to verify these hypotheses, a synthesis model simulating the behavior of the proposed physical model is presented and studied.

\section{SYNTHESIS MODEL}

This section presents a real-time oriented digital scheme allowing to compute the variables of the physical model. This scheme is a straightforward modification of that proposed by Guillemain et al. ${ }^{15}$ using Digital Impedance Models (dimensionless Kirchhoff's variables instead of wave variables) to represent numerically the whole physical model.

The dimensionless variables $\tilde{p}_{g}, \tilde{u}_{g}, \tilde{p}_{m}, \tilde{u}_{m}$ and $\tilde{p}_{r}, \tilde{u}_{r}$ are defined from the physical variables by the relation:

$$
\tilde{p}_{g, m, r}=\frac{p_{g, m, r}}{p_{M}} \quad \tilde{u}_{g, m, r}=Z_{c} \frac{u_{g, m, r}}{p_{M}}
$$

where $p_{M}$ is the static beating-reed pressure involving the physical parameters of the reed and is classically defined by: $p_{M}=\mu_{r} H \omega_{r}^{2}$.

\section{A. Instrument bore}

Using dimensionless variables, the input impedance $Z_{r}(\omega)$ given by equation (3) is written as:

$$
\tilde{Z}_{r}(\omega)=\frac{\tilde{P}_{r}(\omega)}{\tilde{U}_{r}(\omega)}=j \tan (k(\omega) L)=\frac{1-e^{-2 j k(\omega) L}}{1+e^{-2 j k(\omega) L}}
$$

The delay, dispersion and dissipation contained in $\exp (-2 j k(\omega) L)$ are modelled by a first order low-pass digital filter and an integer delay $D=E\left(2 F_{s} L / c\right)$ :

$$
\exp (-2 j k(\omega) L) \simeq \frac{b_{0} z^{-D}}{1-a_{1} z^{-1}}
$$

where $E(x)$ denotes the integer part of $x, F_{s}$ is the sampling frequency and $z=\exp \left(j \omega / F_{s}\right)$.

The coefficients $b_{0}$ and $a_{1}$ are expressed analytically ${ }^{15}$ as functions of the length and radius of the bore by imposing that the height of the first two impedance peaks of the digital model matches those of the continuous model.
This finally leads to the following equations:

$$
\begin{aligned}
\tilde{p}_{r}(n) & =\tilde{u}_{r}(n)+V_{r} \\
V_{r} & =a_{1}\left(\tilde{p}_{r}(n-1)-\tilde{u}_{r}(n-1)\right) \\
& -b_{0}\left(\tilde{p}_{r}(n-D)+\tilde{u}_{r}(n-D)\right)
\end{aligned}
$$

\section{B. Player bore}

The delay and losses contained in $\exp \left(-j k_{m} L_{m}\right)$ are also modelled by a first order low-pass digital filter and an integer delay:

$$
\exp \left(-j k_{m} L_{m}\right) \simeq \frac{b_{m} z^{-D_{m}}}{1-a_{m} z^{-1}}
$$

The delay $D_{m}$ corresponds to $D_{m}=E\left(F_{s} L_{m} / c\right)$. The coefficients $b_{m}$ and $a_{m}$ are computed analytically so that the modulus of the digital model matches, for two given frequencies, that of the continuous model. For an easier control of the model, the first matched frequency is zero, so that the DC component of $p_{g}$ can be entirely transmitted to $p_{m}\left(\tilde{P}_{m}(0)=\tilde{P}_{g}(0)\right)$, yielding $b_{m}=1-a_{m}$. The second frequency is $c /\left(4 L_{m}\right)$ and corresponds to the frequency of the first impedance peak of the player bore.

With this approximation, the time domain digital version of the system of equations (4) and (5) becomes:

$$
\begin{aligned}
\tilde{p}_{g}(n) & =\lambda \tilde{u}_{g}(n)+a_{m}\left(\tilde{p}_{g}(n-1)-\lambda \tilde{u}_{g}(n-1)\right) \\
& +b_{m}\left(\tilde{p}_{m}\left(n-D_{m}\right)-\lambda \tilde{u}_{m}\left(n-D_{m}\right)\right) \\
\tilde{p}_{m}(n) & =-\lambda \tilde{u}_{m}(n)+a_{m}\left(\tilde{p}_{m}(n-1)+\lambda \tilde{u}_{m}(n-1)\right) \\
& +b_{m}\left(\tilde{p}_{g}\left(n-D_{m}\right)+\lambda \tilde{u}_{g}\left(n-D_{m}\right)\right)
\end{aligned}
$$

where $\lambda=Z_{m} / Z_{c}=S / S_{m}$.

Since $\tilde{p}_{g}$ is imposed, equation (18) is modified so that $\tilde{u}_{g}$ can be calculated from $\tilde{p}_{g}, \tilde{p}_{m}$ and $\tilde{u}_{m}$.

This leads to the final set of equations describing the player bore:

$$
\begin{aligned}
\tilde{u}_{g}(n) & =\frac{\tilde{p}_{g}(n)-V_{g}}{\lambda} \\
\tilde{p}_{m}(n) & =-\lambda \tilde{u}_{m}(n)+V_{m}
\end{aligned}
$$

where

$$
\begin{aligned}
V_{g} & =a_{m}\left(\tilde{p}_{g}(n-1)-\lambda \tilde{u}_{g}(n-1)\right) \\
& +b_{m}\left(\tilde{p}_{m}\left(n-D_{m}\right)-\lambda \tilde{u}_{m}\left(n-D_{m}\right)\right) \\
V_{m} & =a_{m}\left(\tilde{p}_{m}(n-1)+\lambda \tilde{u}_{m}(n-1)\right) \\
& +b_{m}\left(\tilde{p}_{g}\left(n-D_{m}\right)+\lambda \tilde{u}_{g}\left(n-D_{m}\right)\right)
\end{aligned}
$$

\section{Reed motion}

The dimensionless reed model consists in replacing the reed displacement $y(t)$ by $x(t)=y(t) / H$. With this notation, the reed opening equation (11) becomes:

$$
S_{y}(t)=w H \theta(1+x(t))(1+x(t))
$$


and the reed dynamics equation (12) becomes:

$$
\frac{1}{\omega_{r}^{2}} \frac{d^{2} x(t)}{d t^{2}}+\frac{q_{r}}{\omega_{r}} \frac{d x(t)}{d t}+x(t)=e(t)
$$

where $e(t)=\tilde{p}_{r}(t)-\tilde{p}_{m}(t)$ denotes the dimensionless reed excitation.

Equation (25) is discretized by the use of centered differentiation schemes: $j \omega \simeq F_{s} / 2\left(z-z^{-1}\right)$ and $-\omega^{2} \simeq$ $F_{s}^{2}\left(z-2+z^{-1}\right)$. This yields the difference equation:

$$
x(n)=b_{1_{a}} e(n-1)+a_{1_{a}} x(n-1)+a_{2_{a}} x(n-2)
$$

where the coefficients $b_{1_{a}}, a_{1_{a}}$ and $a_{2_{a}}$ are expressed analytically $^{15}$ as functions of $\omega_{r}$ and $q_{r}$.

\section{Nonlinear characteristics}

For the sake of digital efficiency, it is assumed that $w H / S_{m} \simeq 0$ and $w H / S \simeq 0$. In this case, from equations (8) and (10) the dimensionless nonlinear characteristics reads:

$$
\tilde{u}_{r}(n)=W \operatorname{sign}\left(\tilde{p}_{m}(n)-\tilde{p}_{r}(n)\right) \sqrt{\left|\tilde{p}_{m}(n)-\tilde{p}_{r}(n)\right|}
$$

where $W$ represents the reed channel opening:

$$
W=\zeta \theta(1+x(n))(1+x(n))
$$

The parameter $\zeta$ corresponds to the definition by Kergomard $^{16}: \zeta=w H Z_{c} \sqrt{2 /\left(\rho p_{M}\right)}$.

\section{E. Synthesis scheme}

The synthesis scheme presented here calculates at any sample $n$ the values of $\tilde{u}_{g}(n), \tilde{p}_{m}(n), \tilde{u}_{m}(n), \tilde{p}_{r}(n), \tilde{u}_{r}(n)$ and $x(n)$ as functions of their past values and the known blowing pressure $\tilde{p}_{g}(n)$ :

- Calculate $V_{g}, V_{m}, V_{r}$ with equations (22), (23), (16) and let $V=V_{m}-V_{r}$

- Calculate $\tilde{u}_{g}(n)$ with equation (20).

- Calculate $x(n)$ with equation (26).

- Calculate $W$ with equation (28).

- Let $\tilde{u}_{m}(n)=\tilde{u}_{r}(n)$, replace $\tilde{p}_{m}(n), \tilde{p}_{r}(n)$ by their definitions from equations $(21),(15)$ into equation (27) and let $b_{c}=1+\lambda$.

- Solve analytically equation (27), yielding:

$\tilde{u}_{r}(n)=\frac{1}{2} \operatorname{sign}(V)\left(-b_{c} W^{2}+W \sqrt{b_{c}^{2} W^{2}+4|V|}\right)$

- Calculate $\tilde{p}_{r}(n), \tilde{p}_{m}(n)$ with equations (15), (21).

- Calculate $e(n)=\tilde{p}_{r}(n)-\tilde{p}_{m}(n)$.

The external pressure $\tilde{p}_{\text {ext }}(n)$ is calculated as the difference between the sum of mouthpiece pressure and flow at sample $n$ and at sample $n-1$, corresponding to the simplest approximation of the derivative of $\tilde{p}_{\text {ext }}(t)$ since:

$$
\tilde{P}_{e x t}(\omega)=j \omega e^{-j k(\omega) L}\left(\tilde{P}_{r}(\omega)+\tilde{U}_{r}(\omega)\right)
$$

where $\exp (-j k(\omega) L)$ can be ignored from a perceptual point of view.

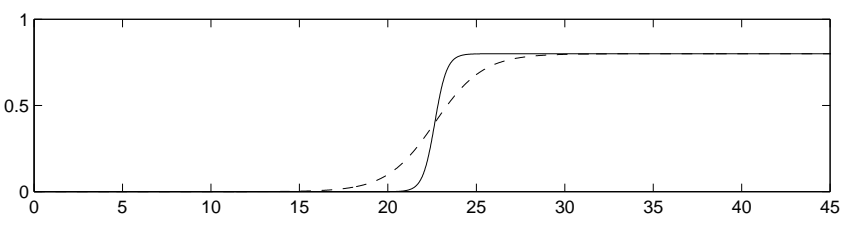

FIG. 7. Typical variations of the transient blowing pressure. Horizontal axis in $m s$.

\section{RESULTS OF SIMULATIONS}

In order to validate the hypothesis made from the analysis of natural sounds, the synthesis model is used to generate sounds.

The transient variations of the blowing pressure $\tilde{p}_{g}(t)$ are controlled as follows:

$$
\tilde{p}_{g}(t)=\frac{\gamma_{c}}{2}\left(1+\tanh \left(\alpha\left(t-t_{0}\right)\right)\right)
$$

The parameter $\gamma_{c}$ controls the pressure level and the parameter $\alpha$ controls the raising time from 0 to the maximum $\gamma_{c}$, therefore the excitation bandwidth. In the model by Kergomard, ${ }^{16} \gamma_{c}$ corresponds to the ratio $p_{m} / p_{M}$. Figure (7) shows typical shapes of the transient pressure for two values of $\alpha$ when $\gamma_{c}=0.8$.

\section{A. Upstream or downstream bore alone}

These examples demonstrate that when the instrument bore is removed, corresponding to impose $\tilde{p}_{r}(t)=0$, self-oscillations can start, tuned on the first peak of an impedance corresponding to the player bore closed by the reed. The reed resonance frequency is chosen high: $f_{r}=10 \mathrm{kHz}$ so that its role on the functioning of the model can be ignored and $q_{r}=0.3$. The values of the control parameters are: $\gamma_{c}=0.8$, corresponding to a beating-reed situation and $\zeta=0.35$. The raising of the blowing pressure is chosen fast: $\alpha=3000 \mathrm{~s}^{-1}$.

\section{Instrument bore alone}

This simulation corresponds to the classical model ignoring the vocal tract. The length of the bore is $L=$ $0.57 \mathrm{~m}$, its radius is $R=7 \mathrm{~mm}$.

The top of figure (8) shows the first $140 \mathrm{~ms}$ of the external pressure, the bottom its spectrogram over a duration of $0.8 s$ and on the frequency range $[03 k H z]$. Though the blowing pressure $\left(\tilde{p}_{m}(t)=\tilde{p}_{g}(t)\right)$ is high and its raising is fast, the envelope of the external pressure is smooth during the attack. A permanent regime is reached at around $t=100 \mathrm{~ms}$, corresponding to a total transient duration of about $45 \mathrm{~ms}$ (the beginning of the sound is at $t=55 \mathrm{~ms}$ ).

The spectrogram shows that mostly odd harmonics are present. The birth of each harmonic is directly related to its rank. Its frequency seems constant during the transient and its amplitude decreases with respect to its rank. 

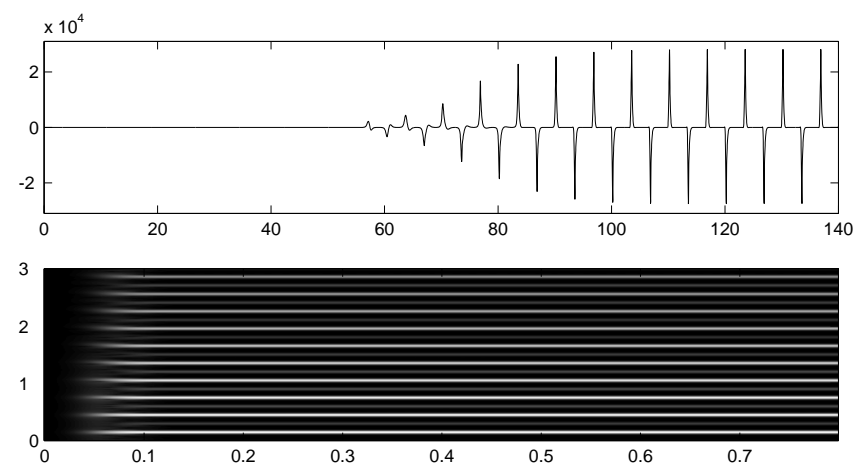

FIG. 8. Instrument bore alone. Top: First $140 \mathrm{~ms}$ of the external pressure. Bottom: Spectrogram on $0.8 \mathrm{~s}$ in the range $(03 k H z)$.

\section{Player bore alone}

This simulation corresponds to the removal of the instrument bore, obtained by setting $\tilde{p}_{r}(t)=0$. The length of the player bore is $L_{m}=0.125 \mathrm{~m}$, its radius is 2.5 times that of the instrument bore: $R_{m}=17.5 \mathrm{~mm}$. The equivalent radius $R_{p}$ used in the calculation of the losses has been chosen five times smaller than the geometrical radius. These values have been adjusted so that the height of the first impedance peak of the player bore be close to that of the third impedance peak of the instrument bore.

The top of figure (9) shows the first $140 \mathrm{~ms}$ of the external pressure, the bottom its spectrogram over a duration of $0.8 s$ and on the frequency range $[03 k \mathrm{~Hz}]$. Compared to figure (8), the raising of the external pressure level nearly follows that of the blowing pressure $\tilde{p}_{g}(t)$ since a steady state regime is reached after the first two periods of oscillations.

The spectrogram shows that mostly odd harmonics with constant frequencies are present. The fundamental frequency of the sound is $650 \mathrm{~Hz}$ and corresponds to that of the first peak of the digital impedance of the player bore. Since the delay $D_{m}$ is quantified, this value differs slightly from that of the first impedance peak of the continuous impedance model which is $c /\left(4 L_{m}\right)=680 \mathrm{~Hz}$.

These two examples show that the functioning of the player/reed and reed/instrument systems is comparable. Indeed, using the notations and results of Kergomard, ${ }^{16}$ by setting $\tilde{p}_{g}=\gamma$ and $\tilde{p}_{g}-\tilde{p}_{m}=p$, it can be shown that for a lossless player bore and a massless reed, in permanent regime $\tilde{p}_{m}$ is a square signal oscillating between 0 and $2 \tilde{p}_{g}$.

Moreover, in order to study the role of the spectral bandwidth of the blowing pressure on the attack duration, the same simulations have been performed with a slower raising of the blowing pressure: $\alpha=200 \mathrm{~s}^{-1}$. These simulations showed that the excitation bandwidth plays a little role on the raising of self-oscillations of the instrument bore alone since the attack duration remained close to $45 \mathrm{~ms}$. On the contrary, a significant influence on the raising of the self-oscillations of the player bore alone
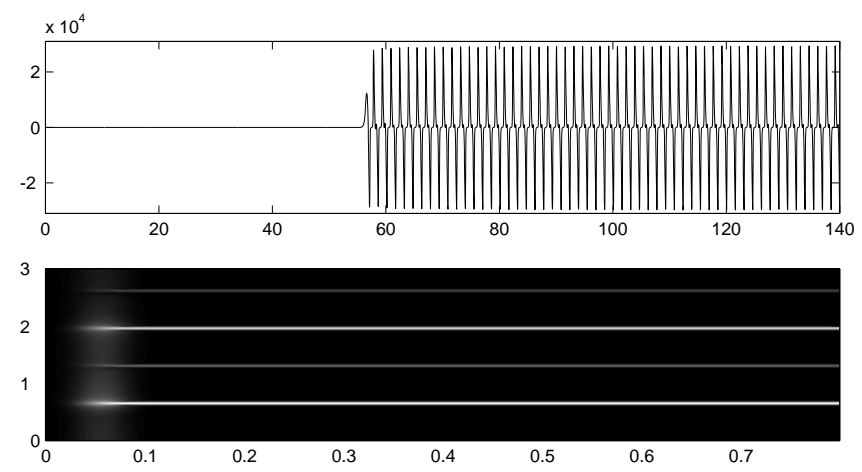

FIG. 9. Player bore alone. Top: First $140 \mathrm{~ms}$ of the external pressure. Bottom: Spectrogram on $0.8 \mathrm{~s}$ in the range $(0$ $3 k H z)$.

was noticed since the steady-state regime was no longer reached instantaneously but after $300 \mathrm{~ms}$.

\section{B. Full model}

This section presents simulations obtained with the full resonator model. The reed resonance frequency has been chosen as $f_{r}=2 k \mathrm{~Hz}$ in order to get closer to a normal playing condition, as it has been measured e.g. by Thompson ${ }^{17}$ and $q_{r}=0.3$. The values of the control and geometrical parameters were adjusted heuristically using the analysis by synthesis concept in order to generate two simulated signals corresponding to the natural examples displayed respectively in figures (3) and (6), according to the hypotheses made in section III B.

\section{Simulation 1}

The first example has been computed with a fast raising of the blowing pressure $\left(\alpha=3000 s^{-1}\right)$. The values of the control and geometrical parameters are: $\gamma_{c}=0.8$, $\zeta=0.35, L=0.57 \mathrm{~m}, R=7 \mathrm{~mm}, L_{m}=0.125 \mathrm{~m}$, $R_{m}=17.5 \mathrm{~mm}, R_{p}=R_{m} / 5$. This set of parameters leads to a height of the first impedance peak of the player bore comparable to that of the third impedance peak of the instrument bore and to a mistuning between the resonances of the player bore and the instrument.

Figure (10) shows, from top to bottom: 1-the mouth pressure in solid line superimposed to the blowing pressure in dashed line; 2-the spectrum of the mouth pressure; 3 -the mouthpiece pressure. The transient duration is $90 \mathrm{~ms}$.

Subplot (1) shows that after an instantaneous raising, the mouth pressure exhibits an oscillating behavior during the first $90 \mathrm{~ms}$. During the first $50 \mathrm{~ms}$ after the beginning, the amplitude of the oscillations remains constant. Then it decays during $40 \mathrm{~ms}$ until, around $t=110 \mathrm{~ms}$, a totally different regime is reached, made of stable oscillations around $\gamma_{c}$.

Subplot (2) shows that the frequency of the transient oscillation is tuned on the first impedance peak of the 

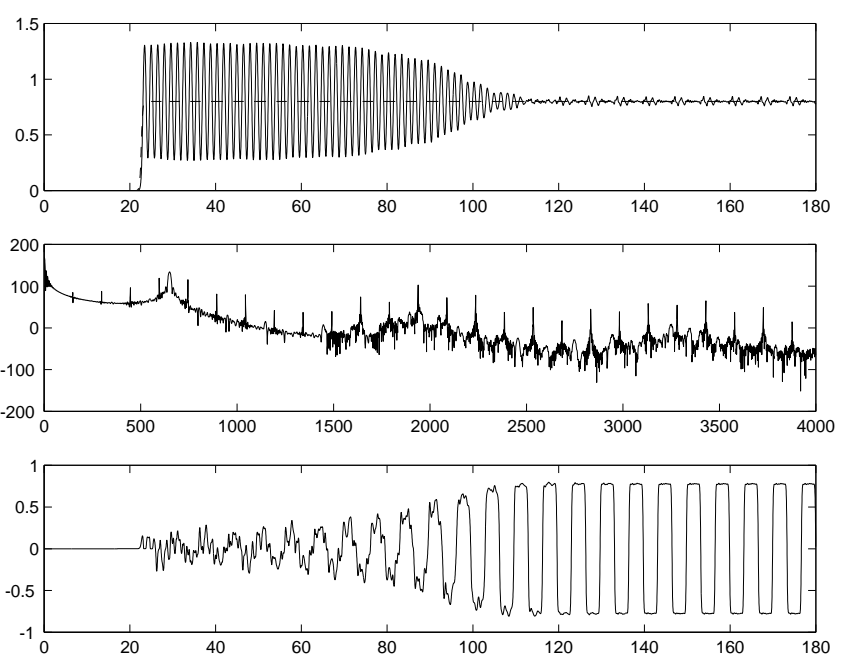

FIG. 10. From top to bottom: 1: First $180 \mathrm{~ms}$ of the mouth pressure (solid line) superimposed to the blowing pressure (dashed line). 2: Spectrum of the mouth pressure in $d B$, horizontal axis in $\mathrm{Hz}$. 3: First $180 \mathrm{~ms}$ of the mouthpiece pressure.

player bore and that the small oscillations of the permanent regime are due to the acoustic coupling of the two bores. Indeed, the spectrum of the mouth pressure clearly shows, at least at high frequency, the sharp (hence not localized in time) harmonics corresponding to selfoscillations of the instrument bore, and a large (hence localized in time) component at $650 \mathrm{~Hz}$.

Subplot (3) shows that the mouthpiece pressure reaches its steady state level at around $t=110 \mathrm{~ms}$. Comparison between subplots (1) and (3) shows that from the beginning of self-oscillations at $t=20 \mathrm{~ms}$, the increasing phase of the oscillations of the mouthpiece pressure corresponds to a decreasing phase of the oscillations of the mouth pressure and that both pressures reach a permanent regime at the same time.

The transient behaviors observed here can be partly explained from the results of the previous subsection. While the mouthpiece pressure remains small, selfoscillations in the mouth, tuned on the first impedance peak of the player bore, starts. These oscillations die when the mouthpiece pressure becomes large enough and a stable regime, tuned on the first resonance of the instrument bore, is reached. Indeed, when the instrument bore is alone, the attack time is $45 \mathrm{~ms}$ while the steadystate regime is reached nearly instantaneously when the player bore is alone. The lengthening of the raising of the mouthpiece pressure (90ms instead of $45 \mathrm{~ms}$ ) could be caused by the oscillations of the mouth pressure, reaching periodically values (around 0.25 ) below the oscillation threshold ${ }^{8}$ of the instrument bore alone (around 0.33).

Figure (11) shows, from top to bottom: 1-the transfer function between the mouthpiece pressure and flow; 2-the transfer function between the mouth pressure and flow; 3 -the equivalent impedance seen from the reed calculated
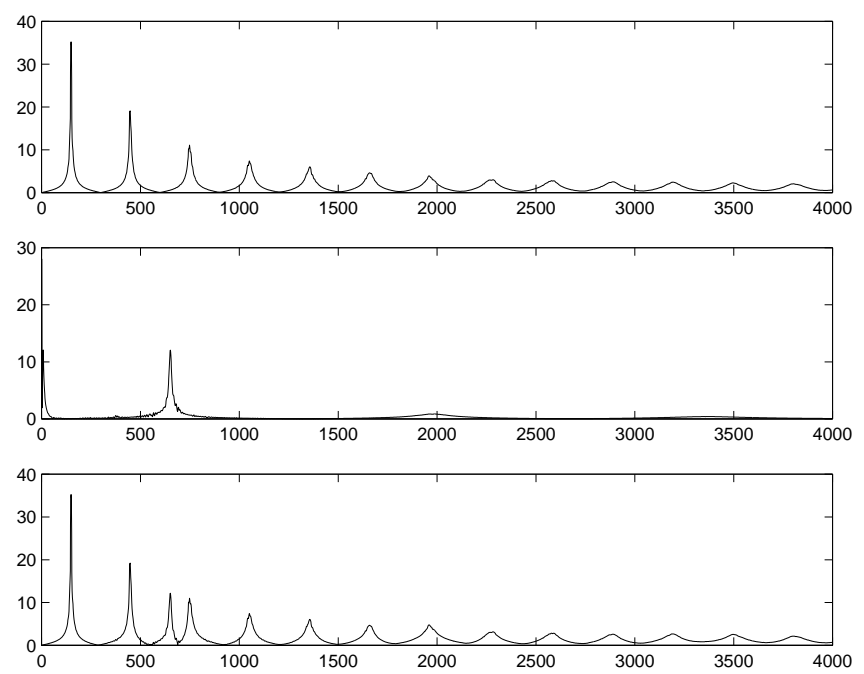

FIG. 11. From top to bottom: 1: Transfer function between mouthpiece pressure and flow; 2: Transfer function between mouth pressure and flow. 3: Equivalent impedance seen from the reed. Horizontal axes in $\mathrm{Hz}$.

as the transfer function between $\tilde{p}_{r}(t)-\left(\tilde{p}_{m}(t)-\tilde{p}_{g}(t)\right)$ and the flow $\tilde{u}_{r}(t)$, corresponding to equation (2).

Subplots (1) and (2) show that the ratio $\tilde{P}_{r}(\omega) / \tilde{U}_{r}(\omega)$ corresponds to the input impedance of the instrument bore alone (the two curves superimpose perfectly) and that the ratio $\tilde{P}_{m}(\omega) / \tilde{U}_{r}(\omega)$ contains a peak at $0 H z$ corresponding to the DC component of the supplied pressure and a peak at $650 \mathrm{~Hz}$ corresponding to the first impedance peak of the player bore. Up to this frequency, this behavior is similar to Scavone' ${ }^{5}$ model and differs at high frequency, with the presence of other peaks, due to the use of a distributed element rather than lumped elements to model the vocal tract.

Subplot (3) shows that the first peak corresponding to the player bore is slightly higher than the third peak corresponding to the instrument bore and that these peaks are not tuned.

The top of figure (12) shows the first $180 \mathrm{~ms}$ of the external pressure, the bottom two vertical slices of the spectrogram displayed on figure (13). Until $t=90 \mathrm{~ms}$, the external pressure exhibits a complex behavior. After $t=90 \mathrm{~ms}$, it becomes similar to the top of figure (8). The two vertical slices of the spectrogram, computed at $t=0.07 \mathrm{~s}$ in solid line and at $t=0.7 \mathrm{~s}$ in dashed line show that during the attack, the component at $650 \mathrm{~Hz}$ and its odd harmonics are visible and vanish totally in the steady state regime.

These behaviors can also be observed on figure (13), which shows that during the first $0.1 s$ of the attack, the spectral content of the sound is rich and inharmonic. Comparison with figure (3) shows many common features and most of the comments of figure (3) remains valid. On the natural sound, the transient component at $690 \mathrm{~Hz}$ appears before the fifth harmonic of the permanent regime while they appear simultaneously on the 

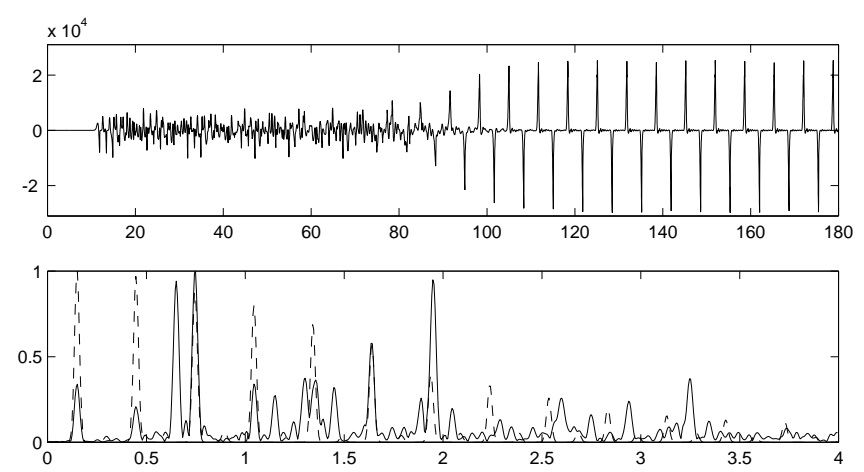

FIG. 12. Top: First $180 \mathrm{~ms}$ of the external pressure. Bottom: Spectrogram slices at $t=0.07 \mathrm{~s}$ (solid line) and $t=0.7 \mathrm{~s}$ (dashed line). Horizontal axis in $k \mathrm{~Hz}$.

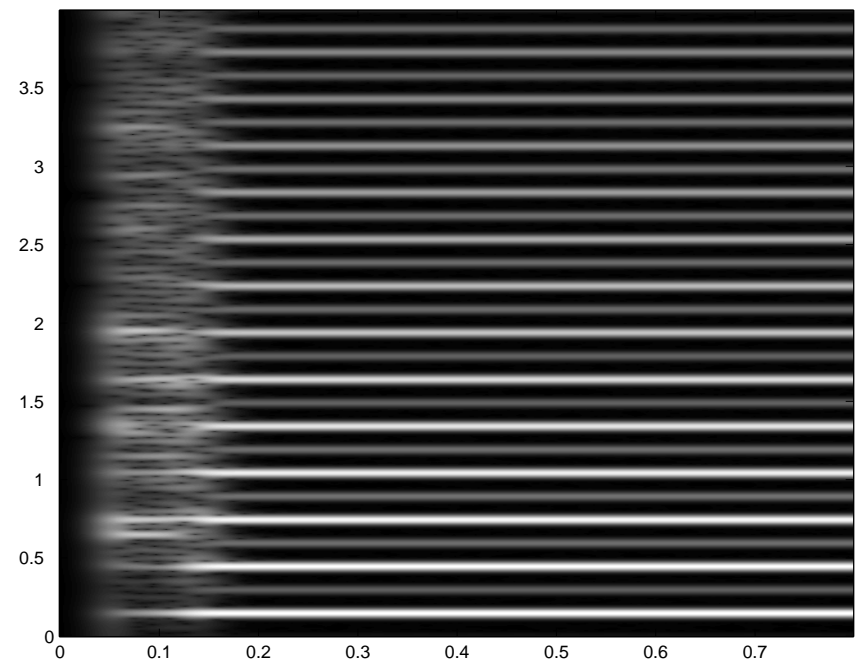

FIG. 13. Spectrogram on $0.8 s$ in the range $(04 k H z)$ corresponding to a fast raising of the blowing pressure.

simulation. The difference in the levels of the transient components between natural and simulated sounds might be attributed to an additional radiation source. Simulations performed with a different reed resonance frequency indicate that the reed does not play any role on the raising of the self-oscillations tuned on the player bore.

\section{Simulation 2}

This second example has been computed with a slower raising speed of the blowing pressure $\left(\alpha=900 \mathrm{~s}^{-1}\right)$. The values of the control and geometrical parameters are: $\gamma_{c}=0.45, \zeta=0.3, L=0.52 \mathrm{~m}, R=7 \mathrm{~mm}, L_{m}=0.17 \mathrm{~m}$, $R_{m}=9.1 \mathrm{~mm}, R_{p}=R_{m} / 4$. The chosen length $L_{m}$ is that of the vocal tract from the glottis to the mouth. Its first resonance frequency $\left(c /\left(4 L_{m}\right)\right)$ is $500 \mathrm{~Hz}$ and corresponds to that of the first impedance peak of the vocal tract in a neutral position (see e.g. Mathur ${ }^{18}$ ). This set of parameters leads to a height of the first impedance peak of the player bore smaller than those of the instrument
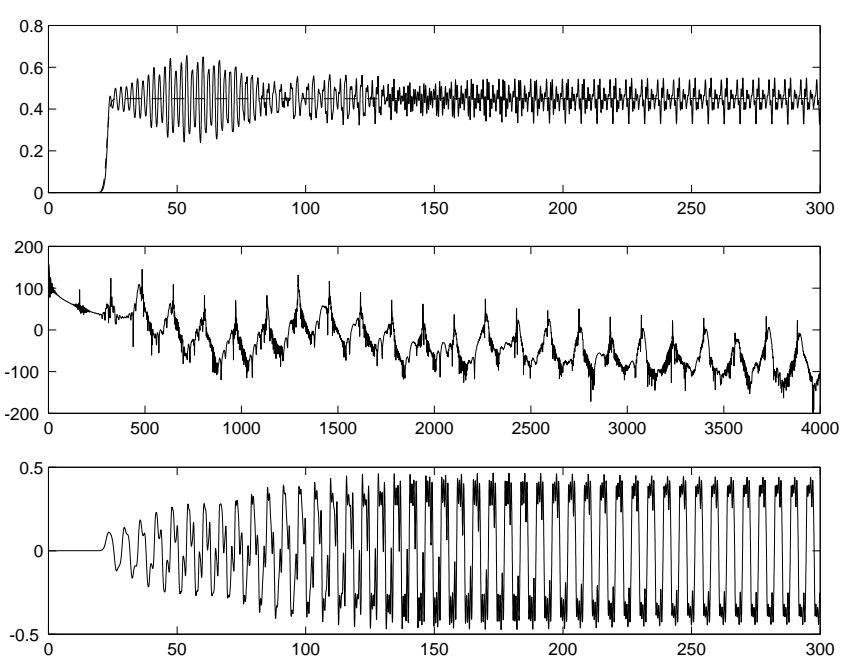

FIG. 14. From top to bottom: 1: First $300 \mathrm{~ms}$ of the mouth pressure (solid line) superimposed to the blowing pressure (dashed line). 2: Spectrum of the mouth pressure in $d B$, horizontal axis in $\mathrm{Hz}$. 3: First $300 \mathrm{~ms}$ of the mouthpiece pressure.

bore and to a strong coupling between the two bores, due to similar radii and to the tuning of the first player bore resonance to the second instrument bore resonance.

Figure (14) shows, from top to bottom: 1-the mouth pressure in solid line superimposed to the blowing pressure in dashed line; 2-the spectrum of the mouth pressure; 3-the mouthpiece pressure. The chosen duration of $300 \mathrm{~ms}$ corresponds to that of the whole transient.

Subplot (1) shows that after a fast raising, the mouth pressure exhibits unstable oscillations until $t=150 \mathrm{~ms}$ that turn into stable oscillations around $\gamma_{c}$. The amplitude of these oscillations is larger than in the first simulation, showing that the coupling between the two bores is more important.

Subplot (2) shows that all the harmonics corresponding to the instrument bore alone are visible. An increase of the level of the harmonics around $500 \mathrm{~Hz}$ can be noticed. The large baselines of all the peaks indicate nonstationary behaviors, such as frequency or amplitude modulations of the harmonics.

Subplot (3) shows that the mouthpiece pressure raises with a step-like shape and exhibits a long nonstationary part, until $t=300 \mathrm{~ms}$.

The top of figure (15) shows the first $300 \mathrm{~ms}$ of the external pressure, the bottom two vertical slices), computed at $t=0.07 \mathrm{~s}$ in solid line and at $t=0.7 \mathrm{~s}$ in dashed line, of the spectrogram displayed on figure (16). Though $\tilde{p}_{g}(t)$ remains constant after its raising, the amplitude of the external pressure shows a complex behavior and reaches a maximum around $t=130 \mathrm{~ms}$. During the transient, the level of harmonic three is higher than that of the others. In the permanent regime, a formant appears around $1200 \mathrm{~Hz}$, with an increase of the level of the harmonics seven, eight and nine. The fundamental frequency of the 

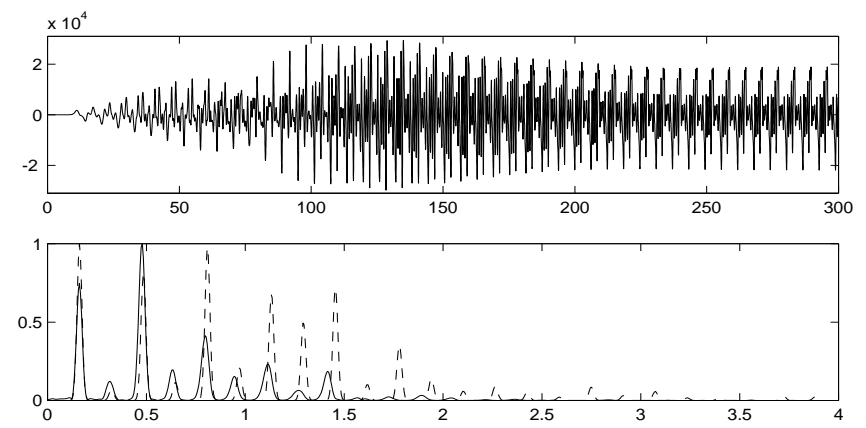

FIG. 15. Top: First 300ms of the external pressure. Bottom: Spectrogram slices at $t=0.07 \mathrm{~s}$ (solid line) and $t=0.7 \mathrm{~s}$ (dashed line). Horizontal axis in $k \mathrm{~Hz}$.

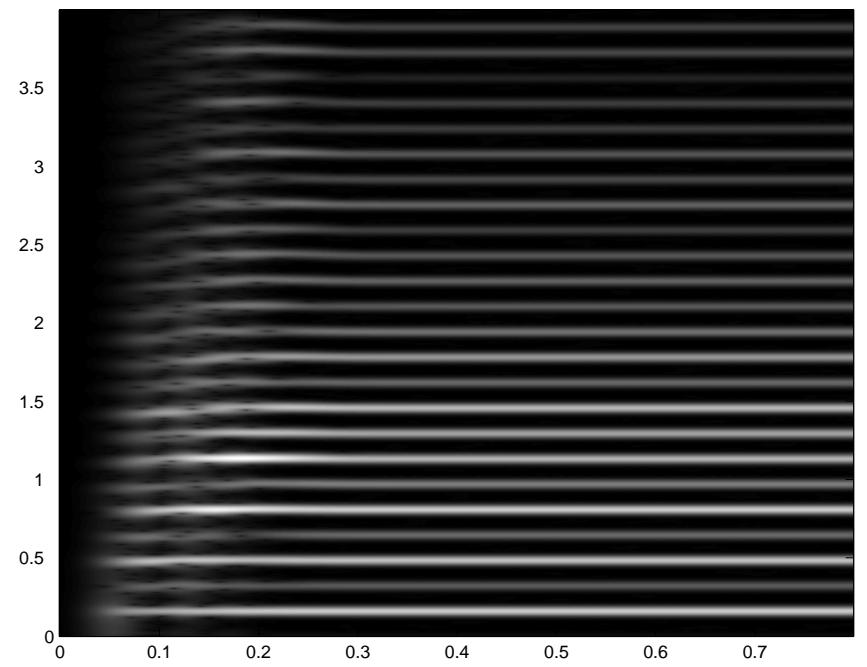

FIG. 16. Spectrogram on $0.8 s$ in the range $(04 k H z)$ corresponding to a slow raising of the blowing pressure.

sound differs during the attack and permanent regimes.

Figure (16) shows significant frequency and amplitude modulations of all the components during the first $0.3 \mathrm{~s}$, as well as a high level of the harmonics five, seven, eight and nine and an early birth of the harmonic three. Most of these features can be linked to those observed in the natural sound example 2 on figure (6). A simulation performed with a reed resonance frequency of $10 \mathrm{kHz}$ indicates that the reed seems to be the main reason of the formant around $1200 \mathrm{~Hz}$ and the player bore the responsible for the frequency modulation and the high level of the harmonics three and five.

\section{CONCLUSIONS}

Thanks to the introduction of a simple physical model of the association player-clarinet and a real-time synthesis scheme, it has been shown that the player's vocal tract might play an important role in some transient situations. Despite its simplicity and low computation cost, the synthesis model allows to generate sounds sharing common features with the natural sounds considered here, both on the transient and the steady-state parts. The "Analysis by Synthesis" concept has been used in order to determine the parameters linked to the vocal tract from the analysis of natural sounds recorded in normal playing conditions. These analyses show that during some attacks, the spectral bandwidth of the pressure transient produced by the player can be high enough to excite a resonance of the respiratory airway, as it is the case in the production of speech consonants.

Simulations using a five cylinders model of the vocal tract did not show any significant difference in the behavior of transient and permanent regimes and further works may rather take into account time-varying geometries or nonlinear effects induced by jets formation at vocal tract constrictions levels and study the role of the vocal tract in the coloring of the breath noise.

Though this study is focused on the clarinet, the same effects would likely be observed and could be simulated on other reed and valve instruments. In particular, large members of the saxophone family for which the reed channel opening is much larger than that of the clarinet and the first impedance peak is low may be even more sensitive to acoustic coupling between the player tract and the instrument bore. In the same way, notes played fortissimo, yielding a high pressure level within the instrument bore, probably increase nonlinear losses in toneholes, yielding a significant lowering of the impedance peaks of the instrument, hence giving even more relative weight to those of the player tract.

Future works will also use a piloted artificial mouth to study experimentally the functioning of the instrument in calibrated transient situations and direct measurements on musicians.

Sound examples are available at:

http://www.lma.cnrs-mrs.fr/ guillemain/JASA06/JASA06.htm

\section{ACKNOWLEDGMENTS}

The author thanks gratefully Claude Crousier, professional clarinet performer and teacher and Richard Kronland-Martinet, member of the Laboratoire de Mécanique et d'Acoustique, for playing the natural sounds presented in this paper.

Jean Kergomard, member of the Laboratoire de Mécanique et d'Acoustique and Claudia Fritz, presently at the Music Faculty of Cambridge University, are deeply thanked for their precious advices.

This work is supported by the "Consonnes" project, funded by the french Agence Nationale de la Recherche.

[1] J. Backus, "The effect of the player's vocal tract on woodwind instrument tone", J. Acoust. Soc. Am., 78(1), 17-20 (1985).

[2] A. Benade, P. Hoekje, "Vocal tract effects in wind instrument regeneration", J. Acoust. Soc. Am. Suppl, 71(1), 591 (1985).

[3] S. Sommerfeldt, W. Strong, "Simulation of a player-clarinet system", J. Acoust. Soc. Am., 83(5), 1908-1919 (1988).

[4] C. Fritz, "La clarinette et le clarinettiste: influence du conduit vocal sur la production du son" (The clarinet and the player: 
role of the vocal tract on the sound production), $\mathrm{Ph}$. D. thesis, Univ. Paris 6 and New South Wales, France (2005).

[5] G. Scavone, "Modeling vocal-tract influence in reed wind instruments", in Proc. 2003 Stockholm Music Acoustics Conference, Stockholm, Sweden (2003).

[6] G. Scavone, "Modeling and Control of Performance Expression in Digital Waveguide Models of Woodwind Instruments", in Proc. 1996 International Computer Music Conference, Hong Kong (1996).

[7] J. C. Risset, "Timbre analysis by synthesis: producing representations, imitations and variants for musical composition", in A. Picciali, G. de Poli and C. Roads, editors, Representations of Musical Signals, M.I.T. Press, Cambridge, Mass., pp. 7-43 (1991).

[8] T. A. Wilson, G. S. Beavers, "Operating modes of the clarinet", J. Acoust. Soc. Am, 56, 653-658 (1974).

[9] M. S. Mukai, "Laryngeal movement while playing wind instruments", in Proc. International Symposium of Musical Acoustics, Tokyo, Japan, pp 239-242 (1992).

[10] H. Levine, J. Schwinger, "On the radiation of sound from an unflanged circular pipe", Phys. Rev., 73(4), 383-406 (1948).

[11] A. D. Pierce, Acoustics, (McGraw-Hill, New York 1981), presently available from Acoust. Soc. Am., New York (1990).
[12] M. M. Sondhi, "Model for wave propagation in a lossy vocal tract", J. Acoust. Soc. Am., 51(6), 1070-1075, (1974).

[13] P. Clinch, G. Troup, L. Harris, "The importance of the vocal tract resonance in clarinet and saxophone performance: A preliminary account", Acustica, Vol. 50, 280-284, (1982).

[14] T. Wilson, "The measured vocal tract impedance for clarinet performance and its role in sound production", J. Acoust. Soc. Am, 99(4), 2455-2456, (1996).

[15] P. Guillemain, J. Kergomard, T. Voinier, "Real-time synthesis of clarinet-like instruments using digital impedance models", J. Acout. Soc. Am, Vol. 118(1), 483-494, (2005).

[16] J. Kergomard, "Elementary considerations on reedinstruments oscillations", in Mechanics of Musical Instruments, edited by Hirschberg et al., Lectures notes CISM, (Springer, New York 1995).

[17] S. C. Thompson, "The effect of the reed resonance on woodwind tone production", J. Acoust. Soc. Am, 66(5), 1299-1307, (1979).

[18] S. Mathur, B. Story, J. Rodriguez, "Vocal-tract modeling: Fractional elongation of segment lengths in a waveguide model with half-sample delays", IEEE Tran. on Audio, Speech and Language Processing, 14(5), (2006). 